\section{Depth evaluation of intended vs actual intacs intrastromal ring segments using optical coherence tomography}

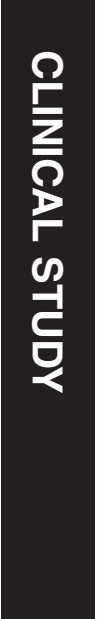

${ }^{1}$ Department of Ophthalmology, Bnai Zion Medical Centre, Haifa, Israel

${ }^{2}$ Hadassah Optimal Private Medical Centre, Affiliated to Hadassah Medical Centre, Haifa, Israel

${ }^{3}$ Ophthalmology Unit, Baruch Padeh Medical Centre, Poriya, Israel

Correspondence: R Barbara, Department of Ophthalmology, Bnai Zion Medical Centre, 29 Tchernikhovski Street, Haifa 35701, Israel Tel: +972052 4448687 E-mail: ramezborbara@ gmail.com

Received: 29 January 2015 Accepted in revised form: 24 August 2015 Published online: 23 October 2015

\begin{abstract}
Purpose Evaluation of actual vs intended intrastromal corneal ring segments (ICRS) implantation depth as measured by anterior segment optical coherence tomography (OCT) Methods Prospective study evaluating 30 Intacs segments implanted manually in 19 eyes of 15 patients suffering from keratoconus. Segment depth evaluation was performed using anterior segment OCT. Measurements were performed above and below the segment at 3 points in relation to the incision site. Statistical analysis was performed using the SAS software for ANOVA, matched $t$-test, and GLIMMIX procedure.

Results Intacs segment depth was $153-\mu \mathrm{m}$ shallower than intended ( $58 \%$ vs $80 \%$ ). Segment layout demonstrated the proximal and distal portions to be $13-\mu \mathrm{m}$ shallower and $12-\mu \mathrm{m}$ deeper (on average), respectively. Intacs segment thickness does not influence implantation depth. Intacs segments implanted in the same eye do not share similar implantation depths. Stromal compression is likely to occur.

Conclusion Intacs are implanted at a shallower depth than intended. The 'pocketing' stage prior to implantation most likely has a stronger effect on the segment's final implantation depth than does the incisions' depth.

Eye (2016) 30, 102-110; doi:10.1038/eye.2015.202; published online 23 October 2015
\end{abstract}

\section{Introduction}

Keratoconus $(\mathrm{KC})$ is a relatively common ectatic degenerative corneal disease. ${ }^{1,2}$ FDA-approved ${ }^{3}$ intrastromal corneal ring segments (ICRS) have been found beneficial for the treatment of
$\mathrm{KC}$ and corneal ectasia following refractive surgery ${ }^{4-6}$ as they flatten and regularize the cornea by means of an arc shortening effect. ${ }^{7}$

The most common types of ICRS used in clinical practice are Intacs (Addition Technology, Inc., Sunnyvale, CA, USA) and Ferrara rings (Ferrara Ophthalmics, Brazil). ${ }^{8}$ Although both are made of polymethylmethacrylate (PMMA), they vary in their radius of curvature and crosssectional shape. The original Intacs segments had $6.77 \mathrm{~mm}$ internal radius, $8.10 \mathrm{~mm}$ outer radius, $250-450 \mu \mathrm{m}$ thicknesses, 150 degrees arc length, and a hexagonal cross-section; ${ }^{9} v s$ $4.4 \mathrm{~mm}, 5.60 \mathrm{~mm}, 200-350 \mu \mathrm{m}, 160$ degrees, and a triangular cross-section respectively for Ferrara rings. ${ }^{8}$ Ferrara rings are hence implanted closer to the centre of the cornea and presumably have a stronger flattening effect. ${ }^{10}$ Intacs SK are a new segment design by Addition Technology, intended for the treatment of moderate to severe keratoconus. They are $6 \mathrm{~mm}$ oval-shaped segments, available in 400 and $450 \mu \mathrm{m}$ thicknesses. ${ }^{2}$ Although it is recommended for ICRS to be implanted at a depth of $66-75 \%$ of the total corneal thickness at the incision site, it is common practice to aim for depths of $70 \%$ for Intacs and $80 \%$ for Ferrara. ${ }^{11}$

There are two methods of implanting ICRS: either femtosecond laser-assisted, or with a mechanical approach. ${ }^{11}$ Shallow implantation is associated with complications such as implant exposure due to corneal thinning over the implant, ${ }^{12}$ implant extrusion, ${ }^{11}$ anterior stromal compression, ${ }^{13}$ compromised diffusion of nutrients to the corneal epithelium, ${ }^{13}$ and segment superficialization. ${ }^{8}$

Assessing ICRS depth may help predict future complications. Slit lamp examination, Pentacam imaging (Oculus Optikgerate GmBH), and anterior segment optical coherence tomography 
(OCT) are common methods for assessing ICRS implantation depth, with the latter two techniques providing the most precise assessment. $8,11,14$

\section{Patients and methods}

This is a prospective study approved by the Helsinki Board at Padeh Medical Centre, Poriya, Israel. All applicable institutional and governmental regulations concerning the ethical use of human volunteers were followed during this research. All patients underwent Intacs ICRS implantation in at least one eye for the treatment of keratoconus. Data collected were segment size, expected segment depth, and actual postoperative ICRS depth as measured by OCT (OPKO Spectral OCT/ SLO) at 3 points for each segment. Thirty intrastromal ring segments implanted in 19 eyes of 15 patients were measured using OCT (OPKO Spectral OCT/SLO). Relevant data including demographics were collected from patients' charts.

\section{Surgical technique}

The surgical procedure was performed by the same surgeon (AB) according to the company's manual and instructional course, ${ }^{9}$ under topical anesthesia. Brieflystandard prepping and draping were performed. The cornea was marked with the special Intacs marker provided by Addition Technology (Lombard, IL, USA). A 1.2-mm radial incision was made along the steep axis at a depth of $80 \%$ of corneal thickness as measured by a pachymeter in the operating room. Pachymetry was done at the incision site and on the hypothetical channels as marked by the special Intacs marker. After applying a semiautomated suction ring and high vacuum by the vacuum system, dissection was performed using a channel guide in clockwise and counterclockwise directions. The Intacs segments were inserted as planned in the created corneal channels. The procedure was sutureless and post operative treatment with topical antibiotics and steroid eye drops were used for 4 weeks.

\section{OCT features}

Three scans were performed for each segment in relation to the incision site. The first, second, and third measurements were at the proximal, central, and distal portions of the segment, respectively. The meridian of the incision and the scan location differed for each patient, as for each eye, the incision was made in the steepest meridian in reference to the corneal topography. The reported variables were calculated as follows:
- Three measurements above the segment were obtained at the proximal, central, and distal portions. Each measurement was performed from the outer corneal surface to the shallow edge of the segment (Figure 1a).

- Three measurements below the segment were obtained at the proximal, central, and distal portions. Each measurement was performed from the inner corneal surface to the inner deep edge of the segment (Figure 1a).

- The averages of the 3 measurements (proximal, central, and distal) above and below the segment were calculated.

- The deepest and shallowest measurements were recorded for each segment. The difference between the maximal and minimal depths for each segment were calculated.

- The percentage depth of segment was calculated by dividing the average reading of the 3 measurements

\section{a}

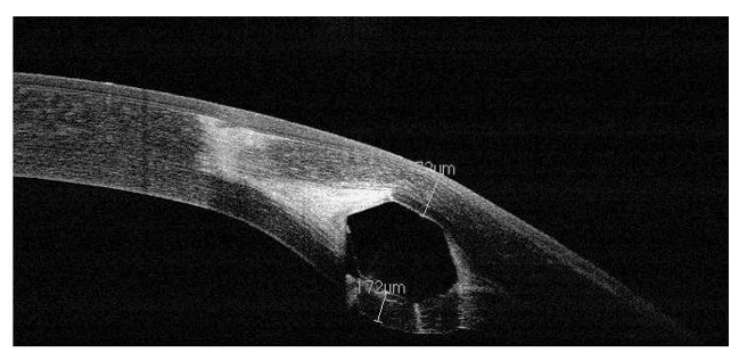

b Distribution of Avg of 3

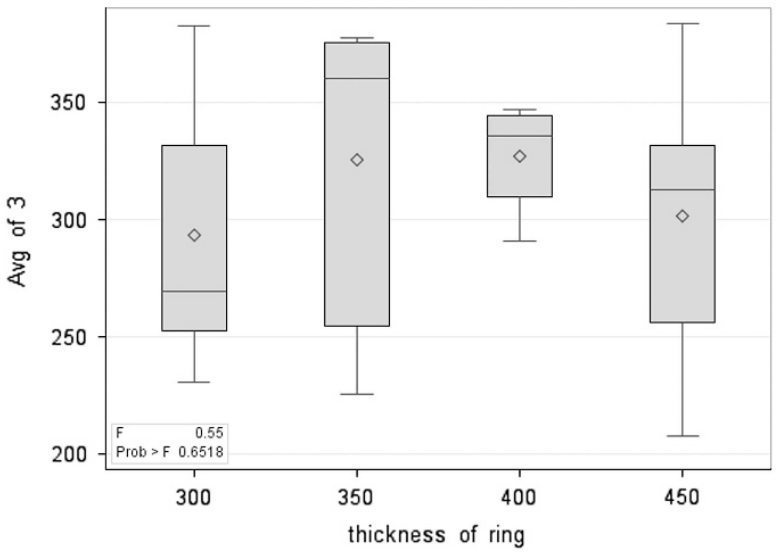

Figure 1 (a) OCT scan of a cornea with ICRS demostrating 2 measurements, above the segment (outer corneal surface to the superficial edge of the segment), and below it (inner corneal surface to the inner deep edge of the segment). (b) Distribution of the average depth values (averages taken of patients' eyes), as a function of segment thickness. These distributions' respective averages are denoted as diamonds, and their medians as horizontal lines. No significant differences were found between the four averages $(F=0.55, P>0.65)$. The graph elucidates the differences in the variances with a relatively small variability when thickness was $400 \mu \mathrm{m}$. 
above the segment by corneal thickness at the thinnest point along the tract (referred to as Method 1): $\left(\frac{\text { Avg }(\text { Proximal }+ \text { Central }+ \text { Distal })}{\text { Thinnest point along the tract }}\right) \times 100$

- The percentage depth of segment (at each of the 3 points) was calculated by measuring the corneal thickness above the segment (TA) in relation to the corneal thickness above (TA) plus the corneal thickness below the segment (TB) as measured by OCT using the formula (referred to as Method 2): $\left(\frac{\mathrm{TA}}{\mathrm{TA}+\mathrm{TB}}\right) \times 100$

\section{Statistical methods}

We applied ANOVA (Analysis of Variance) to compare the means for independent samples, and used the matched $t$-test to estimate and test the differences between the target and the actual values. We applied a mixed model to estimate the intraclass correlation for continuous measurements as the dependent variable, taking into account the data's repeated-measure structure. We applied the GLIMMIX procedure to fit the logistic model with repeated measures. We used the SAS software (SAS Institute, Cary, NC, USA) to perform all the statistical analyses.

\section{Results}

We evaluated 30 segments of 19 eyes of 15 patients.

Table 1 contains all data collected for each segment.

Table 2 summarizes the data.

Patients had one or two segments implanted in each eye depending on clinical need, as judged by the surgeon. Table 3 shows the distribution of the 30 segments. Fifteen segments were $450 \mu \mathrm{m}$ in thickness, four were $400 \mu \mathrm{m}$, six were $350 \mu \mathrm{m}$, and five were $300 \mu \mathrm{m}$ (Table 4).

The thinnest point along the tract was between 504-658 $\mu \mathrm{m}$, with an average of $563 \mu \mathrm{m}$ (SD -59 to +95 ). Incision depth was dependent on corneal thickness at the intended incision site, aiming at $80 \%$ depth and ranging between $420-500 \mu \mathrm{m}$, with an average of $461 \mu \mathrm{m}$ (SD - 41 to +39 ). Corneal thickness at the incision site ranged from 568-687 $\mu \mathrm{m}$, with an average of $607 \mu \mathrm{m}$ (SD -39 to +80 ). Average CCT was $440 \mu \mathrm{m}$ (SD -97 to +110 ; range $343-550 \mu \mathrm{m})$. Average depth of the segments was $295 \mu \mathrm{m}$ (SD -120 to +83 ), $309 \mu \mathrm{m}$ (SD -114 to +96$)$, and $321 \mu \mathrm{m}$ (SD -108 to +125$)$ in the proximal (closest to the incision site), central, and distal portions respectively. Average thickness of the three readings above the segments (proximal, central, and distal) was $308 \mu \mathrm{m}$ (SD - 100 to +70; range 208-378 $\mu \mathrm{m}$ ). The difference between the highest and lowest readings for segment depth was $51 \mu \mathrm{m}$ (SD - 31 to 89 , range $20-140 \mu \mathrm{m}$ ).
Average segment depth calculated using Method 2 was $57 \%, 58 \%$, and $60 \%$ for the proximal, central, and distal portions respectively; the shallowest being 36\%,37\%, and $40 \%$ respectively; and the deepest being $75 \%, 75 \%$, and $82 \%$ respectively. Average corneal thickness below the segment was $227 \mu \mathrm{m}$ (SD -99 to +109$)$, $221 \mu \mathrm{m}$ (SD -100 to +147 ), and $215 \mu \mathrm{m}$ (SD - 114 to 112) for the proximal, central, and distal portions, respectively. Using Method 1 to evaluate segment depth revealed an average of $55 \%$, ranging from $39-69 \%$, which is comparable to the results of Method 2.

As part of our analysis, we aimed to answer the following questions, which might aid in future planning and performing the surgery:

1. What is the probability of two segments implanted in the same eye having similar depths when compared with segments implanted in all eyes?

To answer this question, we fitted a mixed model with the average of the proximal, central, and distal measurements as the dependent variables, taking into account the data's repeated-measures structure. The model indicated a slightly larger variance (1746.54) between the eyes of one patient compared with the variance between the eyes of different patients (1628.92), which yields an intraclass correlation of only 0.48 . Thus we cannot assume that two segments in the same eye will be at closer implanted depths when compared with the segments in other patients' eyes.

\section{Is there a correlation between a segment's thickness and its depth of implantation?}

Although the segments were of differing thicknesses, we still aimed to implant them at $80 \%$ of corneal depth in that area. No correlation was observed between the segment's depth and depth of implantation $(r=-0.03, P=0.87$; Figure $1 \mathrm{~b})$.

\section{What is the statistical significance between a segment's intended and actual depths?}

The mean deviation of the observed compared with the target value is $-152.9 \mu \mathrm{m}$ ( 0.95 confidence interval $(-132.7,-173.1))$, which differs significantly from zero $(P<0.0001)$. This indicates that there is a significantly high probability that the implanted segment will not be as deep as planned (with 0.95 confidence, it will be shallower by more than $132 \mu \mathrm{m})$.

\section{What is the significance of the difference between each measurement above the segment (proximal, central, and distal) and their average?}

We performed calculations for each parameter and compared them to the average readings using the 


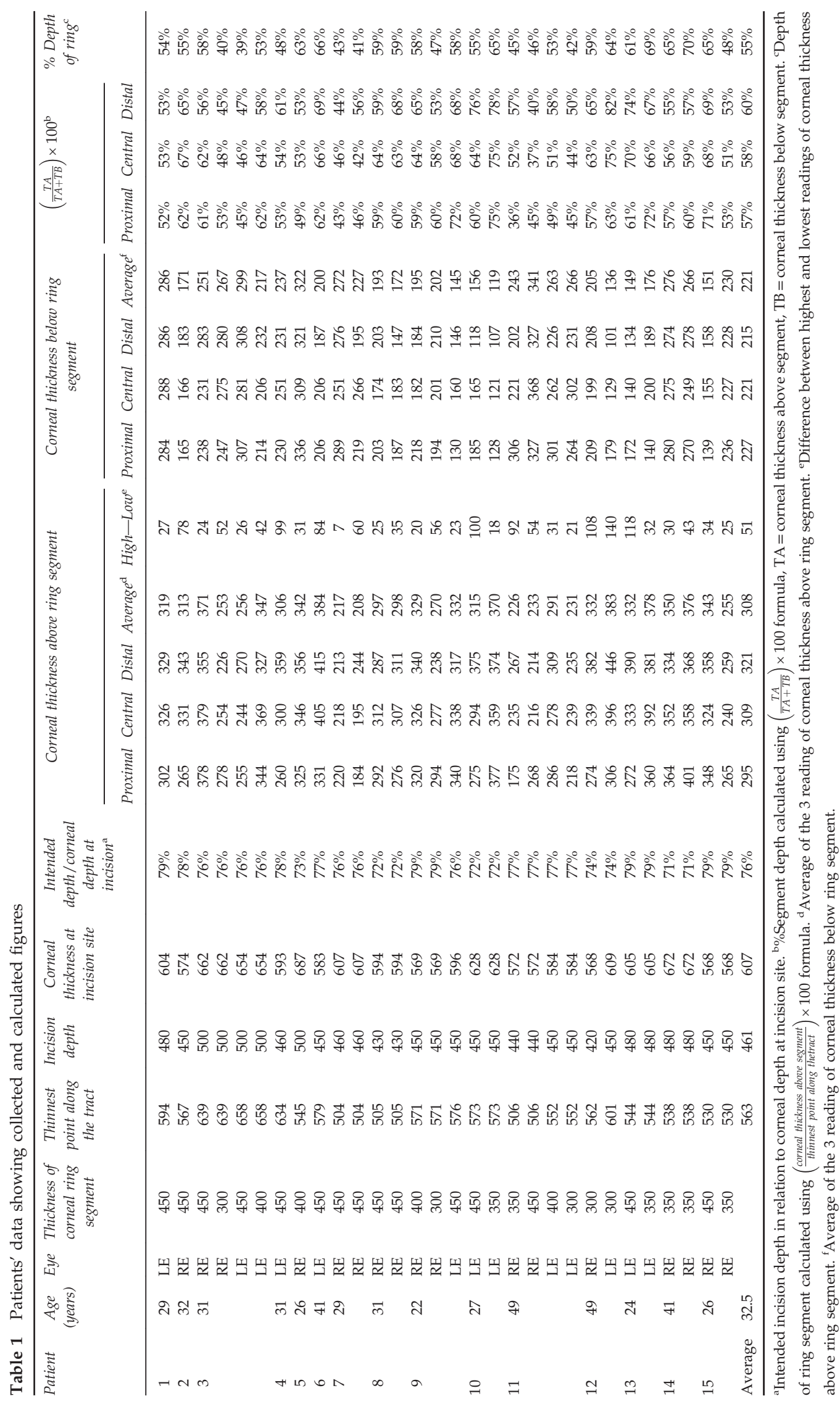


Table 2 Figures and averages of results

\begin{tabular}{|c|c|c|c|c|}
\hline & Range $\mu m$ & Average $\mu \mathrm{m}$ & $\%$ & $S D$ \\
\hline Thinnest point along the tract & $504-658$ & 563 & & -59 to +95 \\
\hline Depth of incision & $420-500$ & 461 & & -41 to +39 \\
\hline Corneal thickness at incision site & $568-687$ & 607 & & -39 to +80 \\
\hline Central Corneal Thickness & $343-500$ & 440 & & -97 to +110 \\
\hline Difference between attempted and actual segments depth & $66-252$ & 153 & & -87 to +99 \\
\hline Difference between highest \& lowest readings for segments depth & $7-140$ & 51 & & -44 to +89 \\
\hline \multicolumn{5}{|l|}{ CTA } \\
\hline Proximal & $175-401$ & 295 & & -120 to +83 \\
\hline Central & $195-405$ & 309 & & -114 to +96 \\
\hline Distal & $213-446$ & 321 & & -108 to +125 \\
\hline Average of CTA (proximal, central \& distal) & $208-384$ & 308 & & -100 to +76 \\
\hline \multicolumn{5}{|l|}{ CTB } \\
\hline Proximal & $130-327$ & 227 & & -99 to +109 \\
\hline Central & $121-309$ & 221 & & -100 to +147 \\
\hline Distal & $101-327$ & 215 & & -114 to +112 \\
\hline Average of CTB (proximal, central \& distal) & 119-322 & 221 & & -102 to +101 \\
\hline \multicolumn{5}{|l|}{$\frac{C T A}{C T A+C T B}$} \\
\hline Proximal & $36-72 \%$ & & 57 & \\
\hline Central & $37-75 \%$ & & 58 & \\
\hline Distal & $40-82 \%$ & & 60 & \\
\hline
\end{tabular}

Abbreviations: CTA, corneal thickness above segment; СТB, corneal thickness below segment.

Table 3 Distribution of the 30 implanted segments in the 19 eyes of 15 patients

\begin{tabular}{lcccc}
\hline Number of patients & $\begin{array}{c}\text { Number of treated } \\
\text { eyes in each patient }\end{array}$ & Total number of eyes & $\begin{array}{c}\text { Number of Intacs } \\
\text { segments in each eye }\end{array}$ & $\begin{array}{c}\text { Total number of Intacs } \\
\text { segments }\end{array}$ \\
\hline 2 & 2 & 4 & 2 & 8 \\
6 & 1 & 6 & 2 & 12 \\
5 & 1 & 5 & 1 & 5 \\
1 & 2 & 2 & 1 & 3 \\
1 & 2 & 2 & & 2 \\
15 patients & & 19 eyes & 30 Segments \\
\hline
\end{tabular}

Table 4 The number of each segment thickness used

\begin{tabular}{cc}
\hline Number of segments used & Thickness of segments $\mu \mathrm{m}$ \\
\hline 15 & 450 \\
4 & 400 \\
6 & 350 \\
5 & 300 \\
\hline
\end{tabular}

Univariate procedure. The estimated expected value of the deviation between the proximal above, and the average is -13.31 ( 0.95 confidence interval $(-24.20$, $-2.41)$ ), which differs significantly from zero $(P=0.0184)$. The estimated expected value of the deviation between the central above and the average is 0.98 ( 0.95 confidence interval $(-3.73,5.71))$, which does not significantly differ from zero $(P=0.67)$. The estimated expected value of the deviation between the distal above and the average is
12.32 (0.95 confidence interval $(2.17,22.47))$, which differs significantly from zero $(P=0.019)$.

\section{What is the significance of the difference between each measurement below the segment (proximal, central, and distal) and their average?}

We performed calculations for each parameter and compared them with the average readings using the Univariate procedure. The estimated expected value of the deviation below the proximal segment and the average was 5.67 (0.95 confidence interval $(-1.9,-13.24))$, which does not significantly differ from zero $(P=0.137)$. The estimated expected value of the deviation between the below the central segment and the average was 0.33 (0.95 confidence interval $(-5.8,6.47))$, which does not significantly differ from zero $(P=0.91)$. The estimated expected value of the 
deviation below the distal segment and the average was $-6.0(0.95$ confidence interval $(-13.3,1.13))$, which does not significantly differ from zero $(P=0.096)$.

\section{Comparison between intended and actual measurement at the 3 points (proximal, central, and distal portion of the segment) and their mean}

Univariate procedure was used. The estimated expected value of the deviation between the target value and the proximal depth was 0.195 (0.95 confidence interval (0.16, $0.23)$ ), which differs significantly from zero $(P<0.0001)$. The estimated expected value of the deviation between the target value and the central depth was 0.176 (0.95 confidence interval $(0.13,0.21))$, which differs significantly from zero $(P<0.0001)$. The estimated expected value of the deviation between the target value and the distal depth was 0.16 (0.95 confidence interval $(0.12,0.20))$, which differs significantly from zero $(P<0.0001)$. The estimated expected value of the deviation between the target value and the mean depth of the 3 measurements was 0.18 (0.95 confidence interval $(0.14,0.21))$, which differs significantly from zero $(P<0.0001)$. Thus, for all measurements, actual depth was significantly shallower than intended.

\section{We hypothesize that when a segment is inserted into the cornea, it results in compression of the superior or inferior corneal lamella}

This hypothesis was tested by adding the corneal thicknesses above (TA) and below (TB) the segment and comparing it to the thinnest point measured along the tract (TT) prior to inserting the segment. If subtracting TA + TB from TT results in a positive value, then one could assume that compression has resulted, while a zero or negative value rejects this hypothesis. In our data, 22 values were positive and 8 were negative. In order to test our hypothesis, we defined a binary variable obtaining the value ' 1 ' if the difference was positive, and ' 0 ' otherwise. We used the GLIMMIX procedure of SAS to fit the logistic model with repeated measures, as there was more than one observation per patient. Among the 30 differences, 8 were negative and 22 positive. According to the fitted model, the estimated probability of a positive difference is 0.73 with a 0.95 confidence interval $(0.45,0.90)$.

The estimated odds ratio for an equal chance of positive vs negative difference differs from 1 , but does not attain statistical significance at the 0.05 level. Thus, we cannot reject the hypothesis that there is an equal chance of a positive us negative difference. Moreover, owing to the repeated measure data structure, the effective sample size was not 30 though we measured 30 differences. If those differences were measured on 30 different patients rather than 15 , then a proportion of $22 / 30$ compared with $8 / 30$ would lead to a highly significant conclusion that supports our hypothesis $(P<0.01)$.

\section{Discussion}

ICRSs are an established, approved surgical management option for keratoconus. Although designed for corneal implantation at a depth of $70-80 \%$, this is rarely achieved, as proven by our study and others. ${ }^{8,13,14}$

Various methods of assessing segment depth using OCT and Pentacam ${ }^{14}$ are referred to in the literature; $8,13,15,16$ including measuring from the anterior corneal surface to the apex of the segment; ${ }^{8}$ and from the anterior corneal surface to the inner ring of the segment. ${ }^{13}$ The method of quantifying segment depth common to all mentioned studies is measurement of the anterior corneal thickness only. This study is unique, as we were also able to measure the corneal thickness below the segment, and hence precisely measure ICRS depth in relation to corneal thickness in that area.

Our study demonstrates that the average measured depth of the implanted segments was $153 \mu \mathrm{m}$ shallower than planned (308 $\mu \mathrm{m}$ instead of the expected $461 \mu \mathrm{m}$; $P<0.0001)$ ). The average ICRS depth was $58 \%$ instead of the planned $76 \%$. Comparing the intended vs actual depth at the three points yields a shallower implantation than intended ( $P<0.0001$ for all three measurements). This is also true when comparing the mean of the three measurements to the intended depth $(P<0.0001)$.

Results in the study by Naftali et $a l^{8}$ are consistent with ours, showing a shallower implantation than intended by $120 \mu \mathrm{m}$. Segment depth was $360 \mu \mathrm{m}$, about $50 \mu \mathrm{m}$ deeper than our results and corresponding to $60 \%$ of corneal thickness, vs 55\% depth in our study using Method 1 and $58 \%$ using Method 2.

Koussai et al compared Intacs implantation using manual and femtosecond laser-assisted implantation, demonstrating shallower implantations than intended in both groups $-76 \mu \mathrm{m}$ in the manual, and $86 \mu \mathrm{m}$ in the laserassisted-concluding that there was no difference between the two techniques with regard to segment depth. Gorgun et al $^{16}$ measured anterior stromal thickness from the apex of the Ferrara segment following femtosecond laser-assisted implantation using OCT. On average, the segments were implanted $97-\mu \mathrm{m}$ shallower than intended.

Lai et al ${ }^{13}$ suggested that shallow implantation can cause anterior stromal compression. Based thereon, ICRSs in our study were implanted deeper than $58 \%$ of corneal thickness due to anterior stromal compression. While we cannot be certain whether ICRSs cause anterior stromal compression only. Our statistical analysis can neither reject nor prove this hypothesis, due to the small sample size. 
Although ICRSs implanted in the same eye shared the same initial incision depth, they tended not to be at similar depths to each other compared with all segments in the study (Intraclass correlation of only 0.48). This fact emphasizes the importance of the 'pocketing' stage-and not incision depth-as a determinant of segments' depth of implantation.

The segment's proximal portion was statistically significantly shallower than average $(13 \mu \mathrm{m} ; P=0.0184)$, the distal portion was statistically significantly deeper than average $(12 \mu \mathrm{m} ; P=0.019)$. This suggests that as we create the tunnel using the dissector, the pockets' proximal portions are shallow; and as we keep on dissecting, we move deeper into the cornea. This result contrasts with those reported by Lai et al, which show the distal portion of the segment's shallower location. ${ }^{13}$ Naftali et $a l^{8}$ demonstrated no significant difference between the proximal, central and distal portions of the segments when using Ferrara rings. When evaluating corneal thickness below the segment, this uneven depth was also apparent. However, this difference did not reach statistical significance.

Assessing segment depth could help predict complications associated with superficial implantation, that is, less than $70-80 \%$ which is rarely achieved. Ferrer et al reported that extrusion was related to shallow implantation and positioning the segment close to the incision site. Shallow implantation has been associated with epithelial breakdown owing to reduced diffusion of nutrients to the epithelium. ${ }^{17}$ When shallow implantation occurs, no surgical intervention is needed as long as the anterior corneal surface is intact. Regular follow up and educating the patient of the potential symptoms of extrusion are important. Even shallow implantation results in improved keratometric and visual outcomes, so usually no other surgical measures are needed to improve visual outcome. If extrusion occurs, removal of the segment can be performed safely through the main incision site, followed by topical antibiotics. Visual and refractive benefits are likely to remain following segment removal due to stromal scarring that occurs while the segment is in situ.

Although this was not the objective of this study, most patients enrolled demonstrated improved visual and refractive outcomes. To date (5 years on) none of the patients had complications associated with shallow implantation, raising the question of how shallow the segment has to be before being prone to complications. It may be that other factors such as eye rubbing have to be present to thin the overlying cornea or promote extrusion of an already superficial segment.

This study proves the importance of pocket dissection as a determinant of segment depth rather than merely incision depth. Therefore, adjustment of the way the dissector is inserted could help achieve deeper dissection. However, this is not crucial to improve visual and refractive outcomes.

There are potential practical learning points for surgeons who perform, or who are considering performing ICRS implantation. Aiming for $80 \%$ incision depth does not guarantee $80 \%$ depth of implantation as the pocket-making stage is the main determinant; this applies to thick and thin corneas. This has also been demonstrated when implanting two segments that share same incision site. Most segments in this study had shallower implantation than planned. Thin corneas at the site of incision and along the tract can result in even shallower implantation, probably due to shallow pocketing. This is likely to be related to the surgeon consciously trying to avoid perforation in a thin cornea. Knowing the above results could encourage surgeons to adjust their technique to enable deeper dissection at the pocketmaking stage.

Photorefractive keratectomy has been performed postICRS implantation to correct residual refractive error, ${ }^{18}$ which will result in an even 'shallower' segment. When concern about progression of keratoconus exists, the above can be combined with corneal collagen cross linking.

Limitations of this study include its small sample size. A larger sample size is needed to assess for anterior stromal compression, and provide definitive answers to some of the questions raised in this study. The use of different devices to measure corneal thickness is another potential source of confounding; ultrasonic (US) pachymetry was used intraoperatively while anterior segment OCT was used postoperatively. We are limited by what instruments are available for intraoperative use and while the patient is supine. US pachymetry is the easiest way to measure corneal thickness over the hypothetical channels. A potential preoperative measurement using anterior segment OCT could have been performed, but it would be difficult to correlate that to the exact hypothetical channels.

There is a potential bias in the preoperative pachymetry measurements as we attempted to measure the thinnest point along the tract. Other areas along the tract may have been thicker and therefore in those areas, the measured segment depth would have been different. We have attempted to tackle this issue by measuring corneal thickness at three different zones of the segment. Furthermore, inter- and intraobserver variability are recognized problems with caliper measurements on OCT imaging.

All procedures were performed by a single surgeon and although most published studies report shallow implantation of segments, the outcomes reported 
in the present study are specific for one surgeon. It could have been of value to compare two surgeons' results. While we found incidentally that shallow implantation did not appear to affect visual or refractive outcomes, further work could involve investigating more specifically the correlation between these variables.

\section{Conclusion}

This study is unique because we were able to measure corneal thickness below the implanted segment, and hence precisely measure ICRS depth in relation to corneal thickness in that area. Although shallow implantations are present in our study as in other studies, novel methods of calculating segment depth have been implemented. On the basis of these results, it may be assumed with 95\% confidence that an ICRS segment will be implanted $\sim 130 \mu \mathrm{m}$ shallower than its intended depth. Segment thickness does not influence implantation depth. The pocketing stage has the strongest influence on segment depth, more so than the initial incision depth, while the thinnest point and corneal thickness along the tract have a role in segment depth. We could not determine whether corneal lamellar compression is present and at what level in relation to the ICRS segment.

\section{Summary}

What was known before

- Intacs Depth: it is recommended for Intacs intra-corneal ring segments to be implanted at $70 \%$ depth of the cornea. Shallower implantation than intended does occur in most cases. Superficial implantation Superficial implantation increases the risk of implant exposure due to corneal thinning over the implant, implant extrusion, anterior stromal compression, compromised diffusion of nutrients to the corneal epithelium, and segment superficialization.

- Anterior segment OCT: Anterior segment OCT is accurate method to assess segments depth of implantation.

\section{What this study adds}

- Depth of implantation: we were able to establish the depth of implantation by measuring corneal thickness above and below the segment. this method has not been described before. Shallower depth of implantation is to be expected (58\%).

- Pocket making stage: pocket making stage is the most crucial step in determining segments depth of implantation and not corneal thickness, incision depth or segments' thickness. two segments implanted in the same eye and share the same incision site and incision depth do not share similar implantation depths. This supports the aforementioned statement. Anterior stromal corneal compression is likely to occur when implanting Intacs segment.

\section{Conflict of interest}

The authors declare no conflict of interest.

\section{Acknowledgements}

We thank Professor Ayala Cohen and Dr Nadia Bordo for their contribution in the statistical analysis and Miriam Erez and Dr Andrew Turnbull for the language editing. This paper was presented at the annual congress of the European Society of Cataract and Refractive Surgeons, London, 2014.

\section{References}

1 Salgado-Borges JM, Costa-Ferreira C, Monteiro M, Guilherme-Monteiro J, Torquestti L, Ferrara P AR JR. Refractive, tomographic and biomechanical outcomes after implantation of Ferrara ICRS in keratoconus patients. Int J Kerat Ect Cor Dis 2012; 1(1): 16-21.

2 Borbara R, Garzozi H BA. Combined intacs SK and corneal collagen cross-linking for the treatment of keratoconus. Int J Kerat Ect Cor Dis 2012; 1(2): 109-116.

3 Schanzlin DJ, Asbell PA, Burris TE, Durrie DS. The intrastromal corneal ring segments. Phase II results for the correction of myopia. Ophthalmology 1997; 104(7): 1067.

4 Colin J. European clinical evaluation: use of Intacs for the treatment of keratoconus. J Cataract Refract Surg 2006; 32(5): 747-755.

5 Alio J, Salem T, Artola A, Osman A. Intracorneal rings to correct corneal ectasia after laser in situ keratomileusis. J Cataract Refract Surg 2002; 28(9): 1568.

6 Ertan A, Colin J. Intracorneal rings for keratoconus and keratectasia. J Cataract Refract Surg 2007; 33(7): 1303-1314.

7 Kymionis GD, Siganos CS, Kounis G, Astyrakakis N, Kalyvianaki MI, Pallikaris IG. Management of post-LASIK corneal ectasia with Intacs inserts: one-year results. Arch Ophthalmol 2003; 121(3): 322.

8 Naftali M, Jabaly-Habib H. Depth of intrastromal corneal ring segments by OCT. Eur J Ophthalmol 2013; 23(2): 171-176.

9 Addition Technology Intacs corneal ring segments for the treatment of keratoconus. International Surgeon Training Manual 2007; 3.1-1-3.1-8.

10 Ertan A, Karacal H, Kamburoğlu G. Refractive and topographic results of transepithelial cross-linking treatment in eyes with intacs. Cornea 2009; 28(7): 719-723.

11 Barbara R, Barbara A. Textbook on Keratoconus - New Insights 2012; 126-162.

12 Kanellopoulos AJ, Lawrence HP, Perry HD, Donnenfeld ED Modified intracorneal ring segment implantations (INTACS) for the management of moderate to advanced keratoconus: efficacy and complications. Cornea 2006; 25(1): 29-33.

13 Lai MM, Tang M, Andrade EMM, Li Y, Khurana RN, Song JC et al. Optical coherence tomography to assess intrastromal corneal ring segment depth in keratoconic eyes. J Cataract Refract Surg 2006; 32(11): 1860-1865.

14 Kamburoglu G, Ertan A, Saraçbasi O. Measurement of depth of Intacs implanted via femtosecond laser using Pentacam. $J$ Refract Surg 2009; 25(4): 377-382.

15 Kouassi FX, Buestel C, Raman B, Melinte D, Touboul D, Gallois A CJ. Comparison of the depth predictability of intra 
corneal ring segment implantation by mechanical versus femtosecond laser-assisted techniques using optical coherence tomography (OCT Visante). J Fr Ophtalmol 2012; 35(2): 94-99.

16 Gorgun E, Kucumen RB, Yenerel NM, Ciftci F. Assessment of intrastromal corneal ring segment position with anterior segment optical coherence tomography. Ophthalmic Surg Lasers Imaging 2012; 43(3): 214-221.
17 Ferrer C, Alió JL, Montañés AU, Pérez-Santonja JJ, del Rio MA, de Toledo JA et al. Causes of intrastromal corneal ring segment explantation: clinicopathologic correlation analysis. J Cataract Refract Surg 2010; 36(6): 970-977.

18 Tan BU, Purcell TL, Nalgirkar A, Ehrenhaus MP, Torres LF, Schanzlin DJ. Photorefractive keratectomy for the correction of residual refractive error with Intacs intrastromal corneal ring segments in place. J Cataract Refract Surg 2008; 34(6): 909-915. 